\title{
Editorial
}

\section{New challenges facing ill health in Gulf war veterans}

Between August 1990 and February 1991, nearly 1 million servicemen and women (670 000 from the United States, 45000 from the United Kingdom and the rest from other nations) representing the United Nations were deployed to the Persian Gulf to oppose the Iraqi invasion of Kuwait. Within months of returning from the Gulf conflict, case reports of veterans complaining of multiorgan, non-specific symptoms began to emerge first in the United States and then the United Kingdom. Since then hundreds of millions of dollars and millions of pounds in research grants have been spent, yet the nature of ill health in Gulf veterans remains controversial.

Methodological flaws hampered early studies based on case reports, highly selected samples, and voluntary registers. These were followed by population based studies representative of the military that provided prevalence data on various indices of health. The main findings from these studies were firstly, Gulf veterans report symptoms more often than appropriate military controls; secondly, there is no evidence of a group of symptoms or conditions that are unique to Gulf veterans; thirdly, hospital admissions and death rates, except for accidents, are not increased in Gulf veterans; and fourthly, the most common symptoms reported are suggestive of mental health conditions - such as fatigue, sleeping problems, low mood, and cognitive difficulties.

Various exposures specific to the Gulf have been postulated: oil fires, pesticides, depleted uranium, vaccinations against biological agents, chemical agents, and combat stress. Several population based studies have now shown that self reported illness is associated with self reported exposures, but the same pattern is found in non-Gulf veterans, and no exposure has yet emerged as the single most important causal factor. ${ }^{1}$

Some investigators have used factor analysis, which considers the question of whether the correlations between a set of reported symptoms can be explained by a few unobservable, latent variables or factors. The pattern of symptom reporting is essentially the same in Gulf and non-Gulf veterans. ${ }^{2}$ Although factor analysis on its own cannot be used to establish whether there is a syndrome, it has played an important part in validating epidemiological findings.

In this issue, Cherry et al, who are the second British research team to use survey methods to study ill health in Gulf veterans, report their findings. ${ }^{3} 4$ They too used a large randomly selected population based sample of Gulf and non-Gulf veterans. They achieved a remarkably high response rate, reflecting the endeavours that the team undertook to trace its subjects.

Their first paper reports on the distribution and characteristics of 95 self reported symptoms. Their main findings are that Gulf veterans reported nearly all the symptoms about twice as often, with greater symptom severity scores, than non-Gulf veterans but the order of symptom prevalence was the same for the two cohorts. This finding is important because it corroborates previous United States, ${ }^{5}$ and United Kingdom studies. ${ }^{6}$

With exploratory factor analysis, they extracted the first seven factors in the Gulf cohort. They labelled these factors psychological, peripheral, neurological, respiratory, gastrointestinal, concentration, and appetite in descending order of the amount of variance explained by each one. Labelling factors in this way aims to summarise those symptoms that have the greater loading on each of them and does not necessarily have any clinical relevance. Six factors had factor scores (a measure of the factor that underlies the symptoms that load onto it) significantly higher in the Gulf cohort, reflecting the increased severity of symptoms. The pattern of symptoms that loaded onto these factors was similar to factors derived from factor analysis in other studies. ${ }^{2}$

This is the first group to publish data from cluster analysis. The aim of this statistical method is to cluster together subjects who have similar clinical profiles. They derived six clusters, each subject being assigned to one cluster. Most subjects in both cohorts were grouped within the first two clusters, which represented the healthiest subjects. The last four clusters progressively defined more ill subjects, and each cluster had almost twice as many Gulf veterans than non-Gulf veterans.

In their second paper, they examine the relation between self reported exposures and factor scores in the Gulf cohorts only. Most exposures were significantly associated with each other but only those with coefficients $>0.2$ were reported. In the univariate analysis, each factor was associated with at least one exposure but after taking account of confounding, the associations that remained significant were between mean symptom severity score and a range of exposures in their three categories of exposures; inoculations and the peripheral factor; pesticides and the peripheral factor; pesticides and neurological factor; receiving medical attention and the gastrointestinal factor; and side effects from nerve agent prophylaxis and the gastrointestinal factor. Handling pesticides was associated with a distribution of pain on mannikins that indicated peripheral neuropathy.

They reported that there was a dose-response relation between the number of inoculations reported and the peripheral factor. However, the proportion of subjects who reported that they held their vaccination records also increased with the number of inoculations. They also found a dose-response effect of the reported number of days of handling pesticides, exposed to oil well fires, using nerve agents, and using insect repellant with various factors. They did not report whether these associations were also present in a non-Gulf cohort. Previous studies have shown that the associations between exposures and ill health are not specific to Gulf veterans, despite some exposures generally considered to be specific to the Gulf conflict. ${ }^{6}$ Without a comparison group, it can be difficult to make certain interpretations of the data.

Despite the sound epidemiological and statistical methods in these papers, important methodological obstacles limit the interpretation of these and previous findings, and will continue to hamper future research. The authors consider the limitations of their study thoroughly. They think that self reported measures of ill health and exposures are unlikely to be subject to important recall bias. The activity of completing questionnaires and sociocultural factorssuch as media interest and peer group social networkscould endorse symptoms that were of minor importance or 
distress to the veteran. Gulf veterans may differentially recall exposures compared with non-Gulf veterans because of the continued interest in "Gulf war syndrome". ${ }^{7}$ The authors suggest that these biases are unlikely as they found a dose-response effect but this can also be subject to reporting bias as the reported number of inoculations seemed to relate to the proportion of subjects who reported that they held their records. There were no valid objective measures of exposures at the time of the Gulf conflict and still there are none.

The investigators suggest that the peripheral and neurological factors and the mannikin pain variable and their associations with inoculations and exposure to pesticides may lend some support for a peripheral neuropathy. The non-specific nature of symptoms in standardised questionnaires, the lack of clinical neurological examination, and biases in symptom and exposure measures suggest that the case for a peripheral neuropathy is unproved. An alternative interpretation of the findings of Cherry et al is that there is no evidence of a syndrome unique to Gulf veterans. This is based on the non-specific range of associations between exposures and symptoms, especially their findings that exposures from each of their categories, and occupational factors (rank and current service status), are all associated with mean symptom severity scores.

Future research needs to focus on the determinants of the excess non-specific ill health in Gulf veterans, with measures which are not solely subjective. Case comparison studies with objective medical and neuropsychological examinations are being carried out in the United Kingdom and United States. Qualitative understanding of individual and social mechanisms that bring about changes in the perception of health after deployment is needed. Use of healthcare facilities by Gulf and non-Gulf veterans needs further examination. Potential immune markers that may reflect previous inoculations need to be explored. The apparent similarities between Gulf war syndrome and the clinical features of previous syndromes after conflict are interesting, especially as the constellation of stressors is usually unique to each conflict. ${ }^{8}$ The civilian literature on perceptions of illness in medically unexplained conditions may provide clues on how symptoms are perceived and attributed in military personnel.

The Gulf war reminded us all that health problems after conflicts still occur despite decreasing combat and increasing peace keeping activities of modern military forces. Health problems should be anticipated after future conflicts. In Italy we are already reading about the "Bosnia syndrome". From an occupational health perspective, strategies to prevent or reduce health problems after conflict need to be developed-such as routine surveillance, risk communication, and keeping systematic medical records. Research into ill health in Gulf veterans was delayed, which may have fuelled various conspiracy theories. Research into health problems after future conflicts should be instigated early.

Liaison Psychiatry, Department of Psychological Medicine, K ISMAIL

Guy's, King's, and St Thomas' School of Medicine,

103 Denmark Hill, London SE24 OAQ, UK

Correspondence to: Dr K Ismail

khalida.ismail@iop.kcl.ac.uk

1 Institute of Medicine. Gulf war and health. Washington, DC: National Academy Press, 2000

2 Wartenberg D, Boyd K, Hallman W, et al. Gulf war syndrome: making sense of factor analyses. Conference on illnesses among Gulf War veterans: a decade of scientific research. Alexandria, Virginia: Research Working Group of the Military and Veterans Health Coordinating Board, 2001.

3 Cherry N, Creed F, Silman A, et al. Health and exposures in United Kingdom Gulf war veterans. Part I: The pattern and extent of ill health. Occup Environ Med 2001;58:291-8.

4 Cherry N, Creed F, Silman A, et al. Health and exposures in United Kingdom Gulf war veterans. Part II: The relation of health to exposure. Occup Environ Med 2001;58:299-306.

5 The Iowa Persian Gulf Study Group. Self-reported illnesses and health status among Gulf War veterans. A population based study. $¥ A M A 1997 ; 277$ : tus among

6 Unwin C, Blatchley N, Coker W, et al. Health of UK servicemen who served in the Persian Gulf war. Lancet 1999;353:169-78.

7 McCauley L, Joos S, Lasarev M, et al. Strategies to assess validity of self-reported exposures during the Persian Gulf war. Environ Res 1999;81: 195-205.

8 Marshall G, Davis L, Sherbourne C. A review of the scientific literature as it pertains to Gulf war illnesses. Vol 4. Stress. Santa Monica, CA: RAND Health, 2000 .

Answers to multiple choice questions on Agents causing occupational asthma by C E Mapp on pages 354-360.

(1) (a) False (b) True (c) False (d) True (e) True

(2) (a) True (b) True (c) True (d) False

(3) (a) True (b) False (c) True (d) True (e) True

(4) (a) True (b) False (c) True (d) True (e) False 